\title{
Gingival recession and labial movement of lower incisors
}

\section{Abstracted from \\ Aziz T, Flores-Mir C.}

A systematic review of the association between appliance-induced labial movement of mandibular incisors and gingival recession. Aust Orthod J 2011; 27: 33-39.

Address for correspondence: Dr Carlos Flores-Mir, Faculty of Medicine and Dentistry, University of Alberta Dentistry, 4051 Dentistry/Pharmacy Centre, University of Alberta, Edmonton, AB T6G 2N8, Canada. E-mail: carlosflores@ualberta.ca

\section{Question: Does orthodontic appliance-induced labial movement of lower incisors increase the risk of gingival recession?}

Data sources Medline, PubMed, Embase, Web of Science, Cochrane Database of Systematic Reviews and reference lists of articles meeting the inclusion criteria.

Study selection To be included, the studies had to be clinical trials with multiple cases, looking at orthodontic treatment involving labial movement of lower incisors and gingival recession in these teeth. Data extraction and synthesis Two authors reviewed all abstracts independently, compared results and reached consensus on inclusion/ exclusion through discussion. Quality assessment of the studies was carried out using one of the authors' own scoring systems.

Results There were six studies with a total of 690 participants. No studies were RCTs, all were retrospective and two had no controls. The outcomes measured were the percentage of teeth and/or patients with recession $>0.5 \mathrm{~mm}$ and proclination of the lower incisors as measured by the angle between the mandibular plane and the long axis of the most prominent incisor. The times the measurements were taken at varied from immediately following appliance removal to eight years after removal. Outcomes were measured using various combinations of lateral cephalograms, dental casts, intra-oral colour photographs and assessment of gingival condition. All six studies found no increased risk of gingival recession after labial movement of the mandibular incisors following orthodontic treatment.

Conclusions There was no association between appliance-induced labial movement of mandibular incisors and gingival recession.

\section{Commentary}

Conflict and controversy are central to issues such as gingival recession and the orthodontic labial movement of lower incisors. For some time now, various authors have tried to establish a correlation between the possible iatrogenic effects of appliance induced lower incisor proclination. The predisposition could be attributable to a thin labial plate of bone and often an aberration of keratinised gingival tissue. To exclude other contributing factors such as predisposing periodontal conditions, smoking and the nature of orthodontic tooth movement would be too simplistic.

The systematic review clearly directs itself towards the association of appliance induced labial movement of lower incisors and gingival recession. A fairly comprehensive search of the electronic databases has been done. It is also to the advantage of the study that they have used the requisite skills of an experienced librarian in carrying out a detailed search of the health sciences database. The selection of 10 abstracts from a total of 34 suggests a stringent inclusion and exclusion criteria. There is an inherent methodological strength to the scoring process used by the authors. Assigning a score and then rating the studies has relevance when the level of evidence available is questionable and none of the included studies is randomised.

The results are interesting and provoke some introspection. All the studies have valid statistical analysis for the data they carry. Six studies deny an association of gingival recession and labial advancement of mandibular incisors during orthodontic treatment. It is apparent that the potential risk of such periodontal sequelae would also be related to the degree of proclination and the mandibular symphisial anatomy. Only two studies have looked at this correlation and the results are not conclusive.

The review arrives at the conclusion that there is no strong evidence to support a hypothesis on labial movement of incisors being linked to increased gingival recession. There are some issues that need to be addressed before one could arrive at an evidence-based decision on this issue. None of the studies has taken into account the type of movement of the incisors, which could range from tipping to translation. Orthodontic tooth movements are complex and usually the resultants are not defined in one dimension, so any labial advancement may have an extrusive or intrusive resultant of force. The clear issue of actually quantifying the pre-treatment gingival recession from a crown height seems flawed. There would be an inherent difficulty in defining this, as one would have to exclude attrition, natural form of the teeth and restorations. The second issue addressed by the authors in the discussion is extremely 
pertinent. The use of pre-adjusted edgewise appliances can inadvertently cause a labial movement and flaring of incisors initially which would then correct when the 3rd order movements express during the later part of treatment. So clearly there is a need to focus on predisposing conditions of the mandibular anatomy, the periodontal status and gingival health of the patient, level of keratinised tissue, pre-existing mucogingival problems and habits such as smoking.

The authors need credit for addressing a host of relevant issues while arriving at the conclusion that there is no strong evidence to support a correlation between appliance induced labial movement of mandibular incisors and gingival recession. The message to the discerning orthodontist is to study the anatomy, the predisposing conditions and then calibrate and titrate the orthodontic appliance to prevent anything but the desired and planned tooth movements. The individual variation of genetics and predisposition needs to be studied and charted carefully, before treatment is initiated. The authors have also highlighted the future directions of research in this issue.

Anmol Kalha

I.T.S Dental College, Hospital \& Research Center Greater Noida, NCR , India

Evidence-Based Dentistry (2013) 14, 21-22. doi:10.1038/sj.ebd.6400917 